\title{
Muerte Cerebral, revisión de tema.
}

\section{Brain death, topic review.}

Pág. 75,83

Recibido: 10-05-2020

Aceptado: 24-06-2020

Dr. Ariel Mendelewicz-Montero ${ }^{1}$

Dr. Ioav Chinchilla Tarnopolsky²

1,2. Médico General, Trabajador independiente, San José, Costa Rica.

\section{RESUMEN}

El término muerte cerebral se entiende como el cese irreversible de las funciones cerebrales incluidas las del tronco encefálico. El determinar el fallecimiento de una persona cuando todavía presenta signos vitales genera gran controversia con respecto al tema de donación de órganos y la fiabilidad del diagnóstico certero. Hasta este momento ninguna prueba de gabinete ha demostrado ser completamente adecuada y aunque son muchos las pruebas que se utilizan mundialmente, ninguna ha sido adoptada dentro de una guía oficial.

\section{PALABRAS CLAVES}

Muerte Cerebral, Muerte de Tronco Encefálico, Neurología, Criterios de Muerte Cerebral.

\section{ABSTRACT}

Brain death is understood as the irreversible cessation of brain functions including those of the brain stem. Determining the death of a person when he still has vital signs generates great controversy regarding the issue of organ donation and the reliability of the accurate diagnosis. So far, no ancillary tests have been shown to be completely adequate, and although many tests are used worldwide, none have been adopted within official guidelines.

\section{KEY WORDS}

Brain Death, Brainstem Death, Neurology, Brain Death Criteria

\section{INTRODUCCIÓN:}

El concepto de muerte cerebral (MC) se define como el cese irreversible de todas las funciones de todo el cerebro, incluido el tronco encefálico10.

La evolución del término deriva de mediados del siglo XX como resultado de observaciones 
de pacientes en las unidades de cuidados intensivos $(\mathrm{UCl})$ que gracias a las nuevas terapias de soporte podrían mantenerse en un estado de coma indefinido3.

La situación anterior conllevo a que la comunidad científica se replanteara el concepto de muerte y a definir cuando un individuo que se encontraba con medidas de soporte había fallecido.

Aunado a lo anterior, el surgimiento de la medicina de trasplantes como cura de enfermedades de otra forma terminales, ha colocado a los pacientes con MC como los donadores predilectos, no sin antes surgir múltiples cuestiones éticas y legales en el proceso14,17. Los profesionales en medicina son quienes están calificados y cuentan con la y experiencia para determinar la MC. Sobre esta profesión recae la obligación ética y moral de lograr un diagnóstico certero12.

A pesar de lo anterior, en muchas ocasiones durante la formación médica el profesional no se familiariza con en el concepto de MC y la naturaleza detrás de su diagnóstico. Es por esto que la presente revisión pretende brindar al profesional en salud un marco de referencia actualizado el cual ayude a familiarizarse con el concepto de MC y fortalecer los conceptos relacionados que consideramos importantes para el ejercicio de la medicina. Adicionalmente que la información provista representa una herramienta para ofrecer guía a los familiares en estas situaciones.

\section{MÉTODOS}

Para la redacción de este artículo se realizó una búsqueda de artículos y publicaciones científicas con las palabras, "muerte cerebral", "coma irreversible", "test de apnea". Se consultó la base de datos PubMed y las siguientes fuentes académicas: Academia Americana de Neurología (AAN), Academia Europea de Neurología (EAN), Federación Mundial de Neurología (WFN), The New England Journal of Medicine (NEJM), The Lancet de Neurología, Elsevier y La Gaceta Diario Oficial de Costa Rica. Se tomó en cuenta únicamente artículos en idiomas inglés y español los cuales estuvieran indexados en revistas digitales con un Digital Object Identifier System (DOI). Todos los artículos incluidos fueron escritos en el período 2015-2020 a excepción de 4 escritos publicados en 1983, 1995, 2010 y 2014. Adicionalmente la información relacionada a muerte cerebral pediátrica y muerte cerebral en el embarazo no fue considerada para este escrito debido a sobrepasar los límites de esta revisión.

En total se recopilaron 25 artículos, de los cuales únicamente se tomaron en cuenta 20. Los 5 artículos excluidos corresponden de forma general a aspectos legales en diferentes centros hospitalarios de Europa, autonomía del paciente y representantes legales y datos relacionados de donantes en MC y la donación de órganos. Todos los cuales no son relevantes para esta redacción.

\section{RESEÑA HISTÓRICA:}

Antes de 1950 la muerte era determinada únicamente basada en criterios cardiovasculares. En los años siguientes, paralelo a los avances en terapia intensiva y ventilación mecánica surgió en 1958 un nuevo estado clínico no visto antes en pacientes de las $\mathrm{UCl}$ quienes se mantenían bajo soporte ventilatorio, el "coma dépassé". Estos pacientes no mostraban datos de actividad cerebral a pesar de existir una función cardiovascular preservada18. El concepto fue presentado por primera vez en 
1959 por P. Mollaret y M. Gourlon durante el Congreso Internacional de Neurología. Hacía referencia a un grupo de 23 pacientes con un estado patológico descrito como "un estado más allá de la forma más profunda del coma" en quienes existía una pérdida total y completa de las funciones vegetativas además de capacidad de relación humana (3).

La situación derivó en un problema ético cuando se hizo evidente que pacientes con lesiones cerebrales no compatibles con la vida, podrían ser mantenidos gracias a las nuevas medidas de soporte en un estado de "coma dépassé" indefinido (12). De forma paralela, mejoras en el campo de medicina de trasplantes permitieron en 1965 realizar exitosamente el primer transplante renal con donador en "coma dépassé"18. La creciente demanda a nivel mundial por órganos para trasplante puso en evidencia la necesidad de establecer criterios diagnósticos de MC los cuales pudieran impulsar los programas de trasplante de órganos sólidos $(4,20)$.

Los primeros esfuerzos para definir la MC comenzaron en 1968 con los Criterios de Harvard, seguidos de la Ley de Determinación Uniforme de la Muerte de los Estados Unidos (UDDA) en 1981 incluyendo la MC como equivalente para el concepto de muerte cardíaca clásico (20).

Este primer consenso determinó como $\mathrm{MC}$ a todo individuo que de acuerdo a los estándares médicos aceptados, ha sufrido $(12,18)$ :

- Cese irreversible de las funciones circulatorias y respiratorias, 0

- Cese irreversible de todas las funciones de todo el cerebro, incluido el tronco encefálico.
Esta promulgación sirvió de base para abordar el problema ético creado a mediados del siglo XX y contestar a la pregunta ¿Cuando se declaraba fallecido? Al paciente que se mantenía con medidas de soporte intensivo (12). En 1995 la AAN publicó la primer guía de práctica clínica incluyendo los parámetros necesarios para la declaración de MC. Dichos parámetros fueron actualizados posterior a una revisión en 2010 . De forma similar en 2008, la Academia del Colegio Real de Médicos del Reino Unido publicó una serie de recomendaciones para la determinación de la $\mathrm{MC}$ en diferentes contextos clínicos $(18,20)$.

\section{FISIOPATOLOGÍA}

Entre las principales causas de MC se tienen: accidente cerebrovascular (AVC), daño neuronal hipóxico-isquémico, daño cerebral secundario a trauma craneoencefálico (TCE), hemorragia cerebral, paro cardiorespiratorio y meningoencefalitis1. Una casuística de pacientes turcos publicada en 2019 incluyó 210 pacientes diagnosticados de MC entre 20122018. Las causas de hospitalización fueron: hemorragia intracraneal espontánea $(43,8 \%)$, AVC isquémico $14,3 \%$, hemorragia intracraneal posterior a cirugía endovascular por aneurisma $11,4 \%$, paro cardíaco $9 \%$, tumor cerebral $8,1 \%$, hemorragia intracraneal traumática $7,6 \%$, AVC isquémico y hemorrágico $3,3 \%$ y otros $2,4 \%$ (8).

Secundario a estos procesos se desarrolla edema cerebral que aumenta la presión intracraneal (PIC) de acuerdo a la hipótesis de Monro-Kellie. Este principio establece que la PIC está determinada por el contenido del espacio intracraneal; principalmente 3 componentes: parénquima cerebral, líquido cefalorraquídeo (LCR) y sangre. De tal forma, se tiene: $\mathrm{PIC}=\mathrm{V}$ cerebro + VLCR + Vsangre. Al

\section{(9) $(1) \Theta$}


estar contenidos en un espacio rígido como la bóveda craneana, el aumento de alguno de los componentes debe ser a expensas de algún otro (9).

En los pacientes con $\mathrm{MC}$, el insulto al sistema nervioso central (SNC) es tal, que en consecuencia se produce importante edema cerebral. El desbalance resultante, hace que el volumen del parénquima cerebral aumente al mismo tiempo en que el LCR y el flujo sanguíneo son reducidos. En tales casos el incremento de la PIC continúa al punto de impedir el flujo sanguíneo arterial. La isquemia resultante es la responsable de la necrosis de los tejidos afectados. El daño neuronal resultante deriva de las fases en las que este proceso ocurre. Una primera lesión relacionada con el tejido infartado y otra zona de "penumbra" aledaña en la cual, a medida que la PIC aumenta la isquemia se agrava y más tejido muere (1).

Aún así, es posible que ciertas neuronas del área de penumbra conserven su función. Se ha propuesto que estas neuronas sobrevivientes, casi siempre ubicadas en en el tallo cerebral e hipotálamo son filogenéticamente más antiguas y metabólicamente menos demandantes que las neuronas corticales siendo más resistentes a los procesos de isquemia (1).

Este principio es contemplado en las guías de la AAN al indicar que la preservación de la función neuroendocrina (secreción de ADH por la neurohipófisis) puede coexistir con la lesión irreversible en hemisferios cerebrales y el tallo encefálico (12).

El suceso final en esta serie eventos culmina con un aumento tal de la PIC, que se produce un hernia transtentorial (11). La compresión resultante del tallo cerebral termina por interrumpir completamente su flujo sanguíneo y compromete gravemente los centro vitales del control cardíaco y centros respiratorios (1).

Criterios diagnósticos clínicos y determinación de MC: El diagnóstico de MC se sustenta en 3 parámetros clave, los cuales se detallan a continuación $(4,13,16)$ :

\section{Estado de coma o falta de respuesta:}

ausencia de respuesta motora cerebral al dolor en todas las extremidades (presión del lecho ungueal y presión supraorbital).

\section{Ausencia de reflejos de tronco encefálico:} reflejos pupilares, movimientos oculares, sensación y respuesta motora facial y reflejos faríngeos.

3. Apnea: Para poder realizar la prueba de apnea, el paciente debe permanecer en un estado euvolémico, con una temperatura central $\geq 36,5^{\circ} \mathrm{C}$, una presión arterial sistólica (PAS) $\geq 90$ $\mathrm{mmHg}$, PCO2 normal (PCO2 arterial $\geq 40$ $\mathrm{mmHg}$ ), PO2 normal y no estar en bypass cardiopulmonar. Una vez cumplidas estas condiciones se procede a realizar la prueba de apnea:

- Conectar un oxímetro de pulso y desconectar el ventilador.

- Administrar O2 al 100\%, 6L/min a nivel de la tráquea (o colocar una cánula a nivel de la Carina).

- Observar atentamente por movimientos respiratorios (excursión abdominal o torácica con volúmenes tidales adecuados).

- Medición del PO2 arterial, PCO2 y pH luego de 8 minutos y reconectar al ventilador. 
- Si no se evidencian movimientos respiratorios y el PCO2 $\geq 60 \mathrm{mmHg}$ (aumento de $\geq 20 \mathrm{mmHg}$ de PCO2 sobre el basal) el resultado de la prueba de apnea se considera positiva (apoya el diagnóstico de MC).

- Si se observan movimientos respiratorios, se considera la prueba de apnea como negativa (no apoya el diagnóstico de $\mathrm{MC}$ ) y se debe repetir el examen.

- Conecte el ventilador si durante la prueba la PAS $\leq 90 \mathrm{mmHg}$, la saturación de oxígeno disminuye de forma considerable o se presentan arritmias cardíacas. Inmediatamente se debe obtener una muestra arterial para análisis de gases. Si la PCO2 $\geq 60 \mathrm{mmHg}$ o aumenta $\geq 20 \mathrm{mmHg}$ sobre el basal se considera una prueba de apnea positiva (apoya el diagnóstico de MC). Si la PCO2 $<60 \mathrm{mmHg}$ o aumenta de $<20 \mathrm{mmHg}$ sobre el basal la prueba de apnea se considera indeterminada $y$ es necesario realizar otras evaluaciones para la confirmación.

A pesar de los cuidados anteriores, ciertas circunstancias pueden interferir con una adecuada valoración clínica: trauma facial o cervical severo, trastornos metabólicos, anomalías pupilares preexistentes, intoxicaciones, apnea del sueño y enfermedad pulmonar severa con retención crónica de CO2. Es posible que bajo estas situaciones se requiera de pruebas confirmatorias que apoyen el diagnóstico. A pesar de lo anterior, la MC continúa definiéndose por parámetros clínicos y el uso de pruebas confirmatorias no es necesario. Estas últimas se relegan como opcionales según el criterio del evaluador y únicamente aconsejables en pacientes en que la valoración convencional no puede realizarse de manera confiable (ver "Pruebas y exámenes confirmatorios").
Múltiples reportes han documentado pacientes en $\mathrm{MC}$ en los que se observa manifestaciones en apariencia contradictorias con el diagnóstico. Algunas de estas ocurrencias pueden explican por preservación aislada de cierta función cerebral (i.e. secreción de $\mathrm{ADH}$ ) aunque la mayoría responden a mecanismos indeterminados. Lo cierto es que su presencia es compatible y no descalifica el diagnóstico de MC.

1. Movimientos espontáneos de las extremidades como el signo de Lázaro (flexión bilateral de los brazos hacia el pecho, aducción del hombro y cruce de la manos) (7) diferentes a las posturas de decorticación o descerebración.

2. Movimientos respiratorios sin cambios significativos del volumen tidal.

3. Sudoración, taquicardia o rubor.

4. Presion arterial (PA) normal, o aumento repentino sin uso de fármacos.

\section{Ausencia de Diabetes Insipida.}

6. Reflejos tendinosos profundos, reflejos abdominales superficiales o respuesta de triple flexión (flexión del tobillo, rodilla y cadera a estímulos en el pie).

7. Reflejo de Babinski.

\section{PRUEBAS Y EXÁMENES CONFIRMATORIOS}

La AAN establece que el diagnóstico de MC solo requiere de parámetros clínicos. A pesar de lo anterior muchos centros alrededor del mundo han incorporado el uso de pruebas diagnósticas de forma rutinaria e incluso obligatoria para determinar la MC. 
El rol de pruebas diagnósticas se relega como opcional según el criterio del evaluador y únicamente aconsejables por la AAN en pacientes en que la valoración convencional no puede realizarse de manera confiable. Las exámenes de gabinete para el diagnóstico de MC pueden dividirse en dos grandes categorías:

\section{Pruebas electrofisiológicas.}

II. Pruebas de perfusión cerebral.

Los resultados reportados son variables y sujetos a interpretación de quien los realiza. Existe una constante discusión internacional a fin de determinar la prueba más sensible y específica, por lo que actualmente no se cuenta con un "Gold Standard". De igual forma la mayoría de los hospitales cuentan con logística necesaria para realizar e interpretar electroencefalograma (EEG). Otras técnicas más avanzadas son pruebas de medicina nuclear y angiografías cerebrales. Estos son los estudios considerados como preferidos (20). A continuación se detallan las características de algunos de ellos:

\section{ELECTROENCEFALOGRAMA}

un método barato y accesible en la mayoría de los centros médicos, ampliamente utilizado en pacientes con sospecha de MC. Consiste en la colocación de electrodos sobre el cuero cabelludo a fin de determinar la actividad eléctrica en las diferentes partes del cerebro.

El objetivo es poder evidenciar la inactividad electrocerebral $(\mathrm{ECl})$ lo cual es sugestivo de MC. Existe gran variabilidad interobservador. Su uso en la $\mathrm{UCl}$ es limitado por la gran cantidad de artefactos que pueden resultar en falsos reportes (4).

\section{POTENCIALES EVOCADOS}

Es una prueba de fácil acceso, permite valorar las vías corticales y del tallo por medio de los potencialessomatosensitivosyauditivos. Poseen la ventaja de ser más resistentes a la presencia de sedantes y a la hipotermia comparado con el EEG y permiten una mejor valoración del tallo y estructuras subcorticales(2).

\section{TOMOGRAFÍA COMPUTARIZADA DE PERFUSIÓN:}

Es una variante de la angiotomografía convencional. Posee una sensibilidad del 100\% comparado con esta última. Esta modalidad de imagen permite demostrar tejido cerebral no viable cuando se demuestra un flujo sanguíneo cerebral $<10 \mathrm{~mL} / 100 \mathrm{~g}$ de parénquima cerebral por min (15). A pesar de estas ventajas su uso todavía es reservado a centro de alta tecnología por la poca disponibilidad de estos tomógrafos.

\section{ULTRASONIDO DOPPLER TRANSCRANEAL}

Es una prueba de fácil acceso, poco costo, y sin exposición a radiación ionizante. Al igual que otras pruebas su agudeza es operador dependiente. El objetivo consiste en evidenciar un paro circulatorio cerebral. Su ventaja sobre métodos como angiografía o medicina nuclear cerebral radica en que es no invasivo y no utiliza medios de contraste que pueden causar obstrucción vascular perjudicial para cualquier función cerebral restante (6).

Regulación costarricense: Actualmente en Costa Rica el concepto legal de MC se define dentro del marco regulatorio de la Ley de Donación y Trasplante de Órganos y Tejidos Humanos (5) No. 9222, vigente desde el año 2014. En ella se establece lo siguiente:

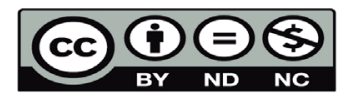


I. Con respecto a la definición de diagnóstico de muerte el artículo 3 Inciso C: Diagnóstico de muerte: como cese irreversible de las funciones circulatorias y respiratorias, cese irreversible de las funciones del cerebro completo, incluyendo las del tronco del encéfalo. Por lo tanto, la muerte puede ser determinada por criterios cardiopulmonares (ausencia de latido cardíaco, ausencia de movimientos respiratorios, ausencia de actividad eléctrica cardíaca efectiva) o por criterios neurológicos (muerte encefálica).

II. Con respecto al diagnóstico y la certificación de la MC el artículo 28: El diagnóstico y la certificación de la muerte encefálica deberán ser reconocidos mediante un examen clínico adecuado tras un período apropiado de observación. Los criterios diagnósticos clínicos, los períodos de observación, así como las pruebas confirmatorias que se requieran, según las circunstancias médicas, serán emitidos por el Ministerio de Salud y de acatamiento obligatorio tanto para el sector público como el privado, y se elaborarán con base en la recomendación del Consejo Nacional de Donación y Trasplante de Órganos y Tejidos.

III. Adicionalmente el artículo 29 establece: El diagnóstico y la certificación de la muerte encefálica serán suscritos por tres médicos del hospital en el que falleció la persona, entre los que debe figurar un neurólogo o neurocirujano y el jefe de la unidad médica donde se encuentre ingresado, o su sustituto. En ningún caso, los médicos que diagnostican y certifican la muerte podrán formar parte del equipo de extracción o trasplante de los órganos que se extraigan.

\section{CONCLUSIONES:}

La MC es un diagnóstico médico que por sus características únicas se deben aplicar criterios estrictos antes de llegar a una conclusión final. El proceso de evaluación es complejo y debe ser realizado por personal capacitado apegándose a las guías clínicas de la AAN. Esta práctica debe realizarse bajo los más altos estándares éticos. La implementación de módulos educativos y el uso de listas de comprobación de requisitos para MC reducirá aún más los casos de mal diagnóstico.

Esta revisión contiene los elementos básicos para proveer a los profesionales de salud de los principales conceptos básicos relacionados a la MC así como el proceso necesario para su determinación. A pesar de requerirse profesionales sanitarios específicos para su diagnóstico, el conocimiento por médicos generales sin duda contribuye a los mejores cuidados de estos pacientes, así como de ofrecer orientación a sus familiares.

A pesar de la amplia la evidencia científica con la que se cuenta actualmente; no es posible determinar cuáles pruebas diagnósticas son las más acertadas para el diagnóstico de MC. Múltiples investigaciones futuras aún son necesarias para definir este "Gold Standard"; o porque no la invención de nuevos métodos diagnósticos. Lo anterior refuerza la importancia de un adecuado diagnóstico basado en la clínica. Conforme nuestro entendimiento de los mecanismos causales de esta entidad avance y los tratamientos de soporte intensivo mejoren, será necesario revisar de forma paralela las definiciones de $M C$ a fin de lograr criterios ajustados a la evidencia más reciente. De igual forma, el aparato legal deberá evolucionar para permitir el mejor desempeño del acto médico; lo anterior suscita otro reto a futuro.

\section{CONFLICTOS DE INTERÉS:}

Los autores declaran ningún conflicto de interés. 


\section{BIBLIOGRAFÍA}

1. Bernat J, Dalle Ave A. Aligning the Criterion and Tests for Brain Death. Cambridge Quarterly of Healthcare Ethics. 2019;28(04):635-641.

2. Dalle Ave A, Bernat J. Inconsistencies Between the Criterion and Tests for Brain Death. Journal of Intensive Care Medicine. 2018;:088506661878426.

3. Goulon M, Babinet P, Simon N. Brain Death or Coma Dépassé. Care of the Critically III Patient. $1983 ;: 765-771$.

4. Koenig M, Kaplan P. Brain death. Handbook of Clinical Neurology. 2019;:89-102.

5. Ley de Donación y Trasplante de Órganos y Tejidos Humanos. San José, Costa Rica: Asamblea Legislativa de la República de Costa Rica; 2014.

6. Li Y, Liu S, Xun F, Liu Z, Huang X. Use of Transcranial Doppler Ultrasound for Diagnosis of Brain Death in Patients with Severe Cerebral Injury. Medical Science Monitor. 2016;22:1910-1915.

7. Moon J, Hyun D. Chronic Brain-Dead Patients Who Exhibit Lazarus Sign. Korean Journal of Neurotrauma. 2017;13(2):153.

8. Mutlu N, Peker T, Acar S, Koca B, Soyal Ö, Titiz A et al. Brain Deaths and Donors in an Education and Research Hospital. Transplantation Proceedings. 2019;51(7):2176-2179.

9. Nordström C, Koskinen L, Olivecrona M. Aspects on the Physiological and Biochemical Foundations of Neurocritical Care. Frontiers in Neurology. 2017;8.

10. Practice parameters for determining brain death in adults: (Summary statement). Neurology. 1995;45(5):1012-1014.

11. Riveros Gilardi B, Muñoz López J, Hernández Villegas A, Garay Mora J, Rico Rodríguez O, Chávez Appendini $R$ et al. Types of Cerebral Herniation and Their Imaging Features. RadioGraphics. 2019;39(6):1598-1610.

12. Russell J, Epstein L, Greer D, Kirschen M, Rubin M, Lewis A. Brain death, the determination of brain death, and member guidance for brain death accommodation requests. Neurology. 2019;92(5):228-232.

13. Salih F, Hoffmann O, Brandt S, Masuhr F, Schreiber S, Weissinger F et al. Safety of apnea testing for the diagnosis of brain death: a comprehensive study on neuromonitoring data and blood gas analysis. European Journal of Neurology. 2019;26(6):887-892. 
14. Sandroni C, D'Arrigo S, Callaway C, Cariou A, Dragancea I, Taccone F et al. The rate of brain death and organ donation in patients resuscitated from cardiac arrest: a systematic review and meta-analysis. Intensive Care Medicine. 2016;42(11):1661-1671.

15. Sawicki M, Sołek-Pastuszka J, Chamier-Ciemińska K, Walecka A, Walecki J, Bohatyrewicz R. Computed Tomography Perfusion is a Useful Adjunct to Computed Tomography Angiography in the Diagnosis of Brain Death. Clinical Neuroradiology. 2017;29(1):101-108.

16. Schiff N, Fins J. Brain death and disorders of consciousness. Current Biology. 2016;26(13):R572-R576.

17. Sheehy E, Conrad S, Brigham L, Luskin R, Weber P, Eakin M et al. Estimating the Number of Potential Organ Donors in the United States. The New England Journal of Medicine. 2020.

18. van Veen E, van der Jagt M, Cnossen M, Maas A, de Beaufort I, Menon D et al. Brain death and postmortem organ donation: report of a questionnaire from the CENTER-TBI study. Critical Care. 2018;22(1).

19. Wahlster S, Wijdicks E, Patel P, Greer D, Hemphill J, Carone M et al. Brain death declaration: Practices and perceptions worldwide. Neurology. 2015;84(18):1870-1879.

20. Wijdicks E, Varelas P, Gronseth G, Greer D. Evidence-based guideline update: Determining brain death in adults: Report of the Quality Standards Subcommittee of the American Academy of Neurology. Neurology. 2010;74(23):1911-1918. 